CPT-99/P.3925

USM-TH-86

\title{
CP-violation in high-energy collisions of polarized protons
}

\author{
Sergey Kovalenko ${ }^{a}$ 円, Ivan Schmidt ${ }^{a}$ and Jacques Soffer ${ }^{b}$ \\ ${ }^{a}$ Departamento de Física, Universidad Técnica Federico Santa María, Casilla \\ 110-V, Valparaíso, Chile \\ ${ }^{b}$ Centre de Physique Théorique, CNRS Luminy, Case 907, F-13288 Marseille \\ Cedex 09, France
}

\begin{abstract}
We study the possibility of a large $\mathrm{CP} / \mathrm{T}$-violation in lepton pair production in the collisions of polarized protons at RHIC-BNL. We propose the single transverse spin asymmetry which quantifies the possible $\mathrm{CP} / \mathrm{T}$ violating effect in this reaction as a sensitive indicator of physics beyond the standard model(SM). We consider two examples of mechanisms beyond the SM which can generate a non-zero asymmetry of this type. They rely on the R-parity violating interactions of supersymmetric models and on phenomenological tensor interactions. We estimate the prospects for observing this effect in future experiments.
\end{abstract}

\section{Introduction}

CP-violation is one of the most disturbing problem of particle physics. Being first observed more than 30 years ago in the neutral kaon system, it has not yet appeared at any other place except, probably indirectly as baryon asymmetry of the universe, in the sense that it cannot be understood without CP-violation.

This situation looks puzzling and inspires great efforts both from theoretical and experimental sides in looking for other CP-violating phenomena (for a recent review see, for instance, [1] and references therein).

In the Standard Model (SM) there is only one source of CP-violation. This is the single complex phase of the Cabibbo-Kobayashi-Maskawa (CKM) matrix

\footnotetext{
${ }^{1}$ On leave of absence from the Joint Institute for Nuclear Research, Dubna, Russia
} 
in the quark sector. Thus, CP-violation takes place only in the charged current flavor changing interactions in the quark sector. The physical CP-violating effect is observable in those processes which involve at least two CKM matrix elements. In cases in which only one matrix element is involved, the complex phase can be rotated away by quark fields redefinition, leaving no physical effect.

Extensions of the SM introduce new complex phases enlarging the realm of $\mathrm{CP}$ - and T-violation [2]. The phenomenological pattern of this $\mathrm{CP} / \mathrm{T}$-violation may significantly differ from the SM picture. In the SM, for instance, there is no room for $\mathrm{CP} / \mathrm{T}$-violation in $K^{0} \rightarrow \pi^{+} \bar{\nu} \mu^{-}$, but once generic scalar and/or tensor interactions with complex coupling constants are introduced, $\mathrm{CP} / \mathrm{T}$-odd effects become possible in this decay [3]. The observation of a non-zero transverse muon polarization $P_{T}^{\mu}$ in this process, would be a good evidence for the violation of CP-invariance beyond the SM. Some theoretical estimates have been given [4] and a new preliminary measurement [5] indicates $P_{T}^{\mu}=-0.0042 \pm 0.0049$ (stat.) \pm 0.0009 (syst.), but the sensitivity is expected to be reduced at the $5 \cdot 10^{-4}$ level.

The best place to look for physics that could exist beyond the SM is in observables that either vanish or are very suppressed in the SM. We will see that this is the case for the CP-violating single spin asymmetry measured in transversely polarized proton-proton collisions, which furthermore is expected to have very small SM background. Our purpose is to present this asymmetry as a powerful indicator for physics beyond the SM, and to estimate its possible value in some examples of SM extensions. Specifically we consider the minimal supersymmetric model with R-parity violation and a phenomenological model with tensor interactions in the quark sector. Therefore this paper deals with the possibility of measuring large $\mathrm{CP}$-violation in high-energy collisions, in processes at a $100 \mathrm{GeV}$ scale, an exciting plausible situation which was already considered a few years ago [6]. Earlier, it was also observed that RHIC might produce CP violating events in heavy-ion collisions [7].

\section{Single transverse spin asymmetry}

A vast spin physics program will be soon under way at the RHIC-BNL facility, operating as a polarized $p p$ collider [8]. Among the very many spin asymmetries which can be measured, let us consider the single transverse spin asymmetry $A_{T}^{ \pm}$ in the reactions $p p \rightarrow l^{+} \nu X, l^{-} \bar{\nu} X$, defined as

$$
A_{T}^{ \pm}=\frac{d \sigma^{ \pm}(\uparrow)-d \sigma^{ \pm}(\downarrow)}{d \sigma^{ \pm}(\uparrow)+d \sigma^{ \pm}(\downarrow)},
$$


where $l=e, \mu, \tau$ and $d \sigma^{ \pm}(\uparrow)\left(d \sigma^{ \pm}(\downarrow)\right)$ is the $l^{ \pm} \nu$ production cross section with one proton beam transversely polarized in the up (down) direction, with respect to the normal to the scattering plane. These cross sections can be computed in the Drell-Yan picture in terms of the dominant quark-antiquark fusion reactions

$$
u+\bar{d} \longrightarrow l^{+}+\nu, \quad d+\bar{u} \longrightarrow l^{-}+\bar{\nu}
$$

For the $l^{+} \nu$ production an explicit form of the asymmetry in (11) becomes

$$
A_{T}^{+}=\frac{\left\langle h_{1}^{u}\left(x_{a}\right) \bar{d}\left(x_{b}\right) \hat{a}_{T}^{u}\right\rangle+\left\langle h_{1}^{\bar{d}}\left(x_{a}\right) u\left(x_{b}\right) \hat{a}_{T}^{\bar{d}}\right\rangle}{\left\langle u\left(x_{a}\right) \bar{d}\left(x_{b}\right)\right\rangle+\left\langle\bar{d}\left(x_{a}\right) u\left(x_{b}\right)\right\rangle} .
$$

The brackets stand for some phase space integrations over the fractions $x_{a}$ and $x_{b}$ of the momenta of the initial protons ( $a$ refers to the polarized proton and $b$ to the unpolarized one), carried by the scattering quark $u$ and antiquark $\bar{d}$, whose distributions are $u(x)$ and $\bar{d}(x)$. Here $h_{1}^{q}(x)$ denotes the transversity distribution for quarks (and antiquarks) [9], which measures the difference of the number of quarks with transverse polarization parallel and antiparallel to the proton transverse polarization; and $\hat{a}_{T}^{q}$ denotes the standard single transverse spin asymmetry (polarization or analyzing power) in the two-body reactions (2), where $q=u, \bar{d}$ and $q=d, \bar{u}$ for the first and the second reactions respectively. As is known [10] this asymmetry can be expressed in terms of helicity amplitudes $\left(\lambda_{1}, \lambda_{2}, \lambda_{3}, \lambda_{4}\right)$ of the reaction $\bar{d}_{\lambda_{1}} u_{\lambda_{2}} \rightarrow \nu_{\lambda_{3}} l_{\lambda_{4}}^{+}$where $\lambda_{i}$ are helicities of the particles involved in the reaction. There are 5 types of these helicity amplitudes which we denote as: $\phi_{1}^{(i)}=(++,++),(--,--) ; \phi_{2}^{(i)}=(++,--),(--,++)$; $\phi_{3}^{(i)}=(+-,+-),(-+,-+) ; \phi_{4}^{(i)}=(+-,-+),(-+,+-)$; and 8 single-flip amplitudes of the type $\phi_{5}^{(i)}=(++,-+),(+-,--), \ldots$. The expression for the asymmetry reads

$$
\hat{a}_{T}^{q}=2 \frac{\operatorname{Im} \sum_{\left\{i_{k}\right\}}\left(\phi_{1}^{\left(i_{1}\right)}+\phi_{2}^{\left(i_{2}\right)}+\phi_{3}^{\left(i_{3}\right)}-\phi_{4}^{\left(i_{4}\right)}\right)^{*} \sum_{i} \phi_{5}^{\left(i_{5}\right)}}{\sum_{\left\{i_{k}\right\}}\left(\left|\phi_{j}^{\left(i_{1}\right)}\right|^{2}+\left|\phi_{j}^{\left(i_{2}\right)}\right|^{2}+\left|\phi_{j}^{\left(i_{3}\right)}\right|^{2}+\left|\phi_{j}^{\left(i_{4}\right)}\right|^{2}+2\left|\phi_{5}^{\left(i_{5}\right)}\right|^{2}\right)} .
$$

The sum over $\left\{i_{k}\right\}$ implies summation of all the amplitudes of each class $\phi_{j}^{\left(i_{k}\right)}$. Recall that one does not observe the polarization of the final leptons, thus their helicity states are not fixed.

In the SM the quark subprocesses (2) are mediated by the s-channel $W^{ \pm}$-boson exchange, as shown in Fig.1(a). For the case of interest $\hat{s}>>m_{q}, m_{l}\left(m_{q}\right.$ and $m_{l}$ are the quark and lepton masses), and the corresponding helicity amplitudes are 
given by

$(+-,--)_{l}=U_{u d} \frac{g^{2}}{2} \frac{m_{l} \sqrt{\hat{s}}}{\hat{s}-M_{W}^{2}} \sin \theta, \quad(+-,-+)=-U_{u d} \frac{g^{2}}{2} \frac{\hat{s}}{\hat{s}-M_{W}^{2}}(1-\cos \theta)$.

Notice that only the first amplitude depends on the lepton mass. Here $g$ is the $S U(2)_{L}$ gauge coupling, $U_{u d}$ is the CKM matrix element, $\hat{s}$ and $\theta$ denote the invariant mass of the lepton pair and the angle between the $u$ quark and the muon 3-momenta in the c.m.s. respectively. As seen from Eq. (4) these two amplitudes alone do not produce any contribution to the CP-asymmetry $\hat{a}_{T}^{q}$. We need some source of a complex phase other than $U_{u d}$. This phase may come from the new interactions beyond the SM.

Below we discuss two mechanisms which could generate non-zero CP-asymmetry in Eq. (田). One is suggested by the supersymmetry (SUSY) and another by a phenomenological model with tensor interactions in the quark sector.

\section{$3 \quad R_{p}$ SUSY mechanism}

We start with the minimal R-parity violating $\left(\not R_{p}\right)$ SUSY model ( $\not R_{p} \mathrm{MSSM}$ ) having the superpotential terms

$$
W_{\not R_{\dot{p}}}=\lambda_{i j k} L_{i} L_{j} E_{k}^{c}+\lambda_{i j k}^{\prime} L_{i} Q_{j} D_{k}^{c}+\mu_{i} L_{i} H_{2} .
$$

in addition to the R-parity conserving terms of the minimal SUSY model(MSSM). In Eq. (6) $L, Q$ stand for lepton and quark $S U(2)_{L}$ doublet left-handed superfields, while $E^{c}, D^{c}$ for lepton and down quark singlet superfields. The coupling constants $\lambda_{i j k}, \lambda_{i j k}^{\prime}$ and the mass parameters $\mu_{i}$ are in general complex numbers and, therefore, they can produce CP-violation. Even if these $\not R_{p}$-parameters are taken real, the complex phases reappear after rephasing the quark and lepton fields, to fix their masses real and to obtain the standard form for the CKM matrix. Let us also notice that in the framework of the R-parity conserving MSSM the only mechanism which can induce the processes (2) at tree level is the ordinary W-exchange in Fig.1(a), barring highly suppressed Higgs-exchange contribution. Thus no new CP-violating phases are introduced by the MSSM in this process compared to the SM case.

The superpotential (6) gives the following Lagrangian terms

$$
\begin{aligned}
& \mathcal{L}_{R_{p}}=\lambda_{i 11}^{\prime} \bar{d} P_{L} u \tilde{e}_{L i}+\lambda_{i j 2}^{*} \bar{\nu}_{i} P_{R} \mu \tilde{e}_{L j}^{*}+\lambda_{i 2 k} \bar{\nu}_{i}{ }_{i} P_{L} \mu \tilde{e}_{R k}^{*} \\
& +\lambda_{21 k}^{\prime} \overline{\mu^{c}} P_{L} u \tilde{d}_{R k}^{*}+\lambda_{i 1 k}^{\prime *} \bar{d} P_{R} \nu_{i}^{c} \tilde{d}_{R k}+\lambda_{i j 1}^{\prime} \bar{d} P_{L} \nu_{i} \tilde{d}_{L j}
\end{aligned}
$$


generating contributions to the processes (2) via the s-channel charged slepton and t-channel down squark exchanges as shown in Fig.1(b,c). The corresponding helicity amplitudes are

$$
\begin{aligned}
(--,--)_{l}^{(i)} & =\sum_{m, n, k} \frac{\lambda_{m 11}^{\prime} \lambda_{n i l}^{*} \tilde{V}_{m k} \tilde{V}_{n k}^{*}}{\hat{s}-M_{\tilde{e}_{k}}^{2}} \approx \eta_{l}^{(i)} \frac{\hat{s}}{\hat{s}-M_{\tilde{e}}^{2}}, \\
(+-,-+)_{l}^{(i)} & =-\zeta_{l}^{(i)} \frac{\hat{s} / 2}{\hat{t}+M_{\tilde{d}_{L}}^{2}}(1-\cos \theta), \\
(+-,--)_{l}^{(i)} & =-\zeta_{l}^{(i)} m_{l} \frac{\sqrt{\hat{s}} / 2}{\hat{t}+M_{\tilde{d}_{L}}^{2}} \sin \theta
\end{aligned}
$$

where $\hat{s}=\left(p_{u}+p_{\bar{d}}\right)^{2}, \hat{t}=\left(p_{u}-p_{l}\right)^{2}$ with $p_{u}, p_{\bar{d}}, p_{l}$ being the 4-momenta of the $u, \bar{d}$ quarks and the final lepton $l$. The indexes $(i), l$ denote the amplitude for the final state neutrino $\nu_{i}$ and charged lepton $l$. We introduced the parameters

$$
\eta_{l}^{(i)}=\sum_{k} \lambda_{k 11}^{\prime} \lambda_{k i l}^{*}, \quad \zeta_{l}^{(i)}=\sum_{k} \lambda_{l 1 k}^{\prime} \lambda_{i 1 k}^{\prime *}
$$

In Eq. (8) $\tilde{V}_{m k}$ is the unitary charged slepton flavor mixing matrix. Analogously the down-squark flavor mixing matrix should be introduced in Eqs. (9), (10). Having complex phases, these matrices could contribute to the CP-asymmetry

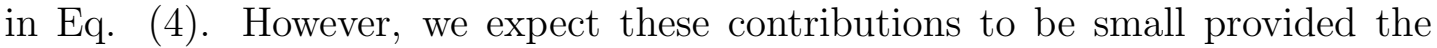
slepton(squark) mass spectrum is nearly degenerate $M_{\tilde{e}_{i}} \approx M_{\tilde{e}}, M_{\tilde{d}_{L}} \approx M_{\tilde{d}_{L}}$, as suggested by the FCNC constrains. Latter on we neglect these mixing matrices as indicated by the approximate equality in (8). The last possible SUSY source of CP-violation in the processes (2) is given by the complex phases of the trilinear soft SUSY breaking parameters $A_{l, d}$ setting the scale of the Higgs-slepton/squark interaction operators $A_{l}\left(H_{1} \tilde{L} \tilde{E}^{c}\right), A_{d}\left(H_{1} \tilde{Q} \tilde{D}^{c}\right)$. The physical effect of these phases manifests itself only through the $\left(\tilde{e}_{L}-\tilde{e}_{R}\right)$ and $\left(\tilde{d}_{L}-\tilde{d}_{R}\right)$-mixing and, therefore, is suppressed by the small factor $m_{e, d} / M_{S U S Y}$. Thus in the framework of the $\not R_{p}$ MSSM a dominant CP-violating effect in the processes (21) is expected to originate from the complex phases $\exp \left(-i \phi_{i k}\right)$ associated with the products of the $\not R_{p}$-couplings in Eqs. (11).

Substituting (5) and (8)-(10) into (4) we obtain in the leading order in small parameters $\eta, \zeta$ the following expression for the CP-asymmetry

$$
\hat{a}_{T(l)}^{u}=-\sin \phi_{C P} r_{l}(\hat{s}) \frac{4 \sin \theta R(\hat{s})\left|\bar{\eta}_{l}\right|}{(1-\cos \theta)^{2}+2 r_{l}^{2}(\hat{s}) \sin ^{2} \theta+4 R^{2}(\hat{s})\left|\eta_{l}\right|^{2}},
$$


with

$$
\begin{aligned}
& \bar{\eta}_{l}=\frac{1}{g^{2}\left|U_{u d}\right|} \sum_{i} \eta_{l}^{(i) *}, \quad\left|\eta_{l}\right|^{2}=\frac{1}{g^{4}\left|U_{u d}\right|^{2}} \sum_{i}\left|\eta_{l}^{(i)}\right|^{2}, \\
& R(\hat{s})=-\frac{\hat{s}-M_{W}^{2}}{\hat{s}-M_{\tilde{e}}^{2}}, \quad r_{l}(\hat{s})=\frac{m_{l}}{\sqrt{\hat{s}}} .
\end{aligned}
$$

Here the summation runs over all the neutrino flavors $\nu_{i}$ in the final state. The angle $\phi_{C P}$ is the relative complex phase between the $U_{u d}$ and the $\not R_{p}$ SUSY parameter $\bar{\eta}$ in Eq. (13). As seen, the contribution of the t-channel diagram in Fig. 1(c) drops out from the leading order expression (12).

Given the suppression factor $m_{l} / \sqrt{\hat{s}}$ the asymmetry in Eq. (12) is larger for the final state $\tau$-lepton in the process (2). For the main muon channel to be studied at the RHIC-BNL experiments this asymmetry is smaller by factor $~$ $6 \cdot 10^{-2}$. It is likely that the tau-channel will also be available in these experiments [13]. First, let us estimate the possible size of the asymmetry (12) for this case. The CP-asymmetry $\hat{a}_{T(\tau)}^{u}$ reaches its maximum value $\left|\hat{a}_{T(\tau)}^{u}\right| \approx 3.4 \cdot \sin \phi_{C P}$ at $\theta \approx 4.5^{\circ},\left|\bar{\eta}_{\tau}\right| \cdot R(\hat{s}) \approx 6 \cdot 10^{-4}$ being practically independent of the individual value of the SUSY parameters $\eta$. Of course, any concrete experiment is limited by the kinematical cut-off $\theta_{\min } \leq \theta \leq \theta_{\max }, 0 \leq R(s) \leq R_{\max }$ which can hardly include this point. Therefore, as an example, let us take a larger angle $\theta=20^{\circ}$. Now the maximum value of the asymmetry

$$
\left|\hat{a}_{T(\tau)}^{u}\right| \approx 0.1 \times \sin \phi_{C P}
$$

is reached at $\left|\bar{\eta}_{\tau}\right| \cdot R(\hat{s}) \approx 0.12$. In order to get the corresponding values of $\hat{s}$ let us estimate the value of the parameter $\bar{\eta}_{\tau}$. From the known constrains on the $R_{p}$ trilinear couplings $\lambda, \lambda^{\prime}$ 14, 15 we can derive the experimental upper bound $\left|\bar{\eta}_{\tau}\right| \leq 7.3 \times 10^{-2}$. According to the above relation this corresponds to $\hat{s} \geq 92 \mathrm{GeV}$, which is not far from the $\mathrm{W}$-pole where maximal statistics is going to be collected in the RHIC-BNL experiments. For the same value of the angle, $\theta=20^{\circ}$, the CP-asymmetry for muon production has the maximum value

$$
\left|\hat{a}_{T(\mu)}^{u}\right| \approx 6 \times 10^{-3} \sin \phi_{C P}
$$

reached at $\left|\overline{\eta_{\mu}}\right| \cdot R(\hat{s}) \approx 0.12$. For this case we have the upper bound $\left|\overline{\eta_{\mu}}\right| \leq$ $3.0 \times 10^{-2}$, derived as in the previous case of tau production. This corresponds to $\hat{s} \geq 96 \mathrm{GeV}$.

The considered mechanism operates out of the W-boson pole since the asymmetry (12) vanishes at $\hat{s}=M_{W}^{2}$. Thus the total event rate is decreased compared to possible mechanisms operating at the $\mathrm{W}$-pole. 


\section{Tensor mechanism}

Let us study an example of the $\mathrm{W}$-pole mechanism in the effective theory with the Lagrangian term [1]

$$
\mathcal{L}_{T}=\frac{1}{\Lambda} \partial_{\nu} W_{\mu}^{-} \bar{d} \sigma^{\mu \nu}\left(f^{L} P_{L}+f^{R} P_{R}\right) u+\ldots,
$$

which may appear at low energies as a footprint of new physics at the scale $\Lambda$. Here $\sigma^{\mu \nu}=(i / 2)\left[\gamma^{\mu}, \gamma^{\nu}\right]$. The lepton sector contains only the SM interactions. A contribution of the operator (16) to the processes (21) is described by the same diagram Fig. 1(a) as in the SM case. The corresponding amplitude is given at $\sqrt{\hat{s}}>>m_{l}, m_{q}$ by the expression

$$
(++,-+)=\frac{g_{2}}{\Lambda} \frac{\sin \theta}{\hat{s}-M_{W}^{2}} \frac{\hat{s}^{3 / 2}}{\sqrt{2}}\left(\left|f_{L}\right|^{2}+\left|f_{R}\right|^{2}\right)^{1 / 2}
$$

which does not depend on the type of the final lepton pair $l \nu$ in the reaction (2). Now we can combine this amplitude with the SM amplitude $(+-,-+)$ in Eq. (5) to calculate a dominant contribution to the asymmetry $\hat{a}_{T}^{u}$. The result is

$$
\hat{a}_{T}^{u}=-2 \frac{\Lambda}{M_{W}} \frac{c r(\theta) \operatorname{Im}\left[U_{u d}^{*}\left(f_{L}+f_{R}\right)\right]}{\left|U_{u d}\right|^{2} c^{2} r^{2}(\theta)\left(\Lambda / M_{W}\right)^{2}+2\left(\left|f_{L}\right|^{2}+\left|f_{R}\right|^{2}\right)},
$$

where $c=\left(2 \sqrt{2} G_{F}\right)^{1 / 2} M_{W}, r(\theta)=\tan ^{-1}(\theta / 2)$. In order to make an estimate of the possible size of this $W$-pole asymmetry we assume that $f_{L} \sim f_{R} \sim f$. Then $\operatorname{Im}\left[U_{u d}^{*}\left(f_{L}+f_{R}\right)\right] \approx 2\left|U_{u d}\right||f| \sin \phi$, where $\phi$ is a relative complex phase of $U_{u d}^{*}$ and $f$. This is a physical phase which can not be removed by the quark and lepton field redefinition. With this approximation we find that the asymmetry in Eq. (18) takes its maximal value

$$
\left|\hat{a}_{T}^{u}\right|_{\text {max }}=\sin \phi \leq 1, \quad \text { at } \quad \tan (\theta / 2)=0.3\left(\frac{\Lambda}{f M_{W}}\right) .
$$

Notice that for $\Lambda /\left(f M_{W}\right) \rightarrow \infty$ the asymmetry reaches its maximal value at the scattering angle $\theta \rightarrow \pi$. If we take $\Lambda \sim M_{W},|f| \sim 1$ then the asymmetry is maximal at $\theta=17^{\circ}$.

\section{Proton level asymmetry}

Now we can estimate the possible size of the proton level asymmetry $A_{T}^{ \pm}$given by formula (3). This quantity, as we argued at the beginning, can be measured in 
polarized proton-proton leptoproduction. For estimation purposes we can significantly simplify formula (3). The second term in the numerator of (3) is certainly

small, since one believes that $h_{1}^{u} \gg h_{1}^{\bar{d}}$ (see ref. [9]). On the other hand, if we select a region where the first term in the denominator dominates (i.e. $x_{a} \gg x_{b}$ ), we end up with

$$
\left\langle A_{T}^{+}\right\rangle \approx\left\langle\frac{h_{1}^{u}}{u}\right\rangle \cdot \hat{a}_{T}^{u}
$$

Using some reasonable theoretical estimates (see ref. [9]) one has $\left\langle h_{1}^{u} / u\right\rangle \approx 0.5$. Therefore we finally obtain

$$
\begin{array}{cll}
\not R_{p} S U S Y: & p p \rightarrow \tau \nu X & \left|\left\langle A_{T}^{+}\right\rangle\right| \leq 5 \times 10^{-2} \text { at } \theta=20^{\circ}, \hat{s}=92 \mathrm{GeV} \\
& p p \rightarrow \mu \nu X & \left|\left\langle A_{T}^{+}\right\rangle\right| \leq 3 \times 10^{-3} \text { at } \theta=20^{\circ}, \hat{s}=96 \mathrm{GeV} \\
\text { Tensors : } & p p \rightarrow l^{+} \nu X & \left|\left\langle A_{T}^{+}\right\rangle\right| \leq 0.5 \text { at } \quad \tan (\theta / 2)=0.3\left(\frac{\Lambda}{f M_{W}}\right) .
\end{array}
$$

These constraints represent certain kinematical samples described in the previous sections which aimed to demonstrate possible size of the CP-asymmetry $\left|\left\langle A_{T}^{+}\right\rangle\right|$. Fore a concrete experiment one has to analyze on the basis of Eqs. (12)-(18) the whole accessible kinematical domain to find out preferable scattering angles and to determine a searching strategy.

The SM backgrounds are expected to be small, due to the fact that the Wproduction vertex involves only one CKM matrix element even at the one-loop level, and in order for the asymmetry to be non-vanishing, several CKM matrix elements, whose relative convention-independent phase is non-zero, have to be present. Higher twist contributions are also expected to be small because they should be suppressed, at least by a factor $M_{\text {proton }} / M_{W}$, in addition to a possible cancellation between the vector and the axial vertices.

\section{Conclusions}

Summarizing, we have shown that there exists the exciting possibility of searching for the $\mathrm{CP}$ violation in polarized p-p collisions. We proposed an appropriate CPasymmetry $A_{T}$ defined in (四) and demonstrated that it may serve as a powerful tool in order to probe new physics beyond the SM. If at a given sensitivity the experiment does not observe the CP-odd effect, setting an upper bound on the asymmetry $A_{T}$, this will still allow one to establish new important upper bounds on the parameters of new physics. 


\section{Acknowledgments}

One of us (JS) would like to thank V. L. Rykov and F. M. Renard for some useful correspondence at the earlier stage of this work, and C. P. Yuan for several enlightening discussions. This work was supported in part by Fondecyt (Chile) under grants 1990806, 1000717, 1980150 and 8000017, and by a Cátedra Presidencial (Chile).

\section{References}

[1] G. Buchalla, Plenary talk given at International Europhysics Conference on High-Energy Physics(EPS-HEP 99), Tampere, Finland, 15-21 July 1999, hep-ph/9912369.

[2] Y. Grossman, Y. Nir and R. Rattazzi, in Heavy Flavours II, eds. A.J. Buras and M. Lindner(World Scientific), hep-ph/9701231; Y. Nir, hep-ph/9911321; G.F. Guidice, hep-ph/9912279.

[3] M. Leurer, Phys. Rev. Lett. 62, 1967 (1989).

[4] J. J. Godina Nava, Phys. Rev. D53, 1703 (1996), and references therein.

[5] E246 KEK-PS Collaboration, A. Ivashkin, talk presented at the EPS-HEP99, Tampere Finland, July 14-21 (1999).

[6] C. J.-C. Im, G. Kane and P. J. Malde, Phys. Lett. B317, 454 (1993).

[7] P. D. Morley and I. Schmidt, Zeit. für Phys. C26, 627 (1985); D. Kharzeev, R. D. Pisarski and M. H. G. Tytgat, Phys. Rev. Lett. 81, 512 (1998).

[8] J. Soffer, Acta Phys. Pol. B29, 1303 (1998), and references therein.

[9] For a recent short review, see J. Soffer, Nucl. Phys. (Proc. Suppl.) 64, 143 (1998).

[10] C. Bourrely, E. Leader and J. Soffer, Phys. Rep. 59, 95 (1980).

[11] G. Kane, G. A. Ladinsky and C.-P. Yuan, Phys. Rev. D45, 124 (1992).

[12] V.L. Rykov, hep-ex/9908050.

[13] Michael Tannenbaum, private communication. 
[14] G. Bhattacharyya, Nucl. Phys. Proc. Suppl. 52 A, 83 (1997); and IFUP-TH 43/97, hep-ph/9709395; H. Dreiner, in Perspectives on Supersymmetry, Ed. by G.L. Kane, World Scientific, 462; P. Roy, hep-ph/9712520; R. Barbier et al., hep-ph/9810232; B.C. Allanach, A. Dedes, H. Dreiner, Phys.Rev. D60 (1999) 075014.

[15] M. Hirsch, H.V. Klapdor-Kleingrothaus and S.G. Kovalenko, Phys.Rev.Lett., 75, 17 (1995); A. Faessler, T.S. Kosmas, S.G. Kovalenko and J.D. Vergados, Nucl.Phys. B587 (2000) 25. 


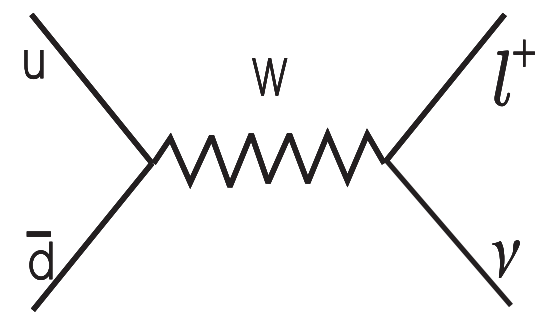

(a)

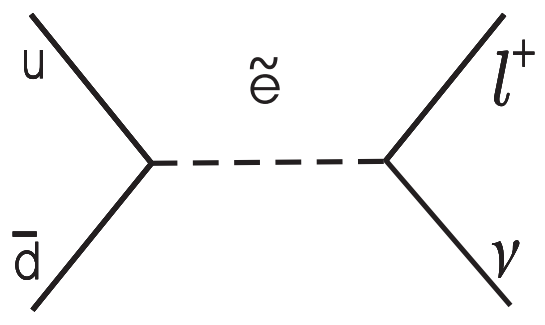

(b)

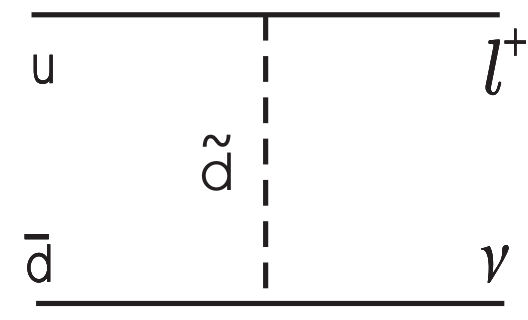

(c)

Figure 1: (a) The standard model W-exchange and (b), (c) the R-parity violating supersymmetric contributions to the Drell-Yan lepton pair production. 